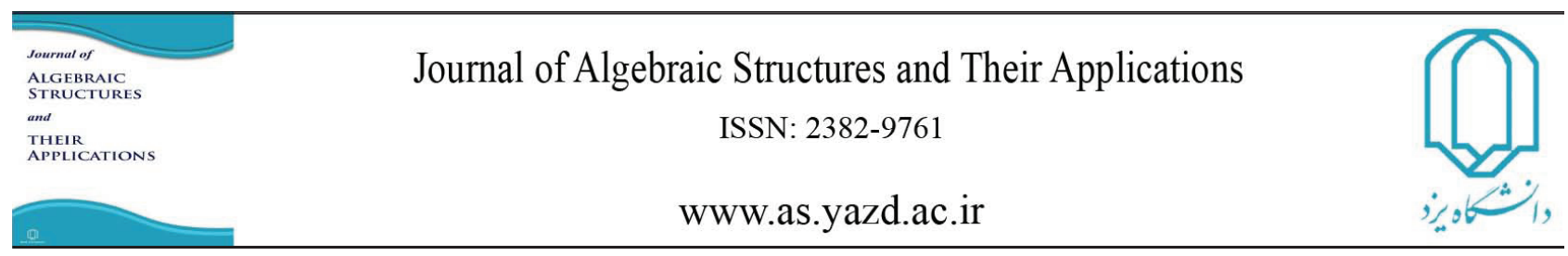

Algebraic Structures and Their Applications Vol. 5 No. 2 ( 2018 ) pp 15-21.

\title{
ON PERMUTABLY COMPLEMENTED SUBALGEBRAS OF FINITE DIMENSIONAL LIE ALGEBRAS
}

\author{
LEILA GOUDARZI
}

\author{
Communicated by S. Alikhani
}

\begin{abstract}
Let $L$ be a finite-dimensional Lie algebra. We say a subalgebra $H$ of $L$ is permutably complemented in $L$ if there is a subalgebra $K$ of $L$ such that $L=H+K$ and $H \cap K=0$. Also, if every subalgebra of $L$ is permutably complemented in $L$, then $L$ is called completely factorisable. In this article, we consider the influence of these concepts on the structure of a Lie algebra, in particular, we obtain some characterizations for supersolvability of a finite-dimensional Lie algebra in terms of permutably complemented subalgebras.
\end{abstract}

\section{INTRODUCTION}

The concept of complemented groups was introduced by Hall [6] and has been studied by a number of authors. For example, Ballester-Bolinches and Guo [T], Christensen [3], Kappe and Kirtland [7].

In $[8]$ Kolman introduced the notion of complemented Lie algebras and this concept was further investigated by Towers [12]. A Lie algebra $L$ is called a complemented Lie algebra, if given

DOI:http://dx.doi.org/10.29252/asta.5.2.15

MSC(2010): 17B05, 17B30, 17B50

Keywords: Lie algebra, permutably complemented, completely factorisable, solvable, supersolvable.

Received: 31 March 2018, Accepted: 19 October 2018.

*Corresponding author

(C) 2018 Yazd University. 
any subalgebra $H$ of $L$ there is a subalgebra $K$ of $L$ such that $H \cap K=0$ and $L=\langle H, K\rangle$. Furthermore, Towers defined the concept of permutable subalgebras in [1:3]. Two subalgebras $A$ and $B$ of $L$ are called permutable, if $\langle A, B\rangle=A+B$. This allows us to define the concept of permutably complemented for a subalgebra of a Lie algebra. We say a subalgebra $H$ of $L$ is permutably complemented in $L$, if there is a subalgebra $K$ of $L$ such that $L=H+K$ and $H \cap K=0$. Also in this case, we say $H$ has a permutable complement in $L$. If every subalgebra of $L$ is permutably complemented in $L$, then $L$ is called completely factorisable. This definition was given by Gein and Mukhin [5]. Also, in [15] Towers gave some properties of completely factorisable Lie algebras. Now, in this paper, we give some characterizations of supersolvability of a finite-dimensional Lie algebra in terms of permutably complemented subalgebras.

A Lie algebra $L$ is called supersolvable if there is a chain $0=L_{0} \subset L_{1} \subset \cdots \subset L_{k-1} \subset L_{k}=L$ such that $L_{i}$ is an $i$-dimensional ideal of $L$.

All Lie algebras referred to in this article are finite-dimensional over a field $F$ of characteristic zero. The Frattini ideal and the nil radical of a Lie algebra $L$ are denoted by $\varphi(L)$ and $N(L)$, respectively. If $H$ and $K$ are subalgebras of $L$, for which $L=H+K$ and $H \cap K=0$ we will write $L=H \dot{+} K$.

\section{Preliminaries}

This section is devoted to some basic results which are needed in our investigation.

Lemma 2.1. Let $L$ be a Lie algebra with subalgebras $H, K$ and ideal $N$.

(i) If $K$ contains $H$ and $H$ is permutably complemented in $L$, then $H$ is permutably complemented in $K$.

(ii) If $N$ is contained in $H$ and $H$ is permutably complemented in $L$, then $H / N$ is permutably complemented in $L / N$.

Proof. Straightforward.

Lemma 2.2. Let $A$ be a minimal abelian ideal of a Lie algebra $L$.

(i) If $A$ has a maximal subalgebra $H$ that is permutably complemented in $L$, then $\operatorname{dim} A=1$.

(ii) If $A$ has a one-dimensional subalgebra $H$ that is permutably complemented in $L$, then $A=H$ and $\operatorname{so} \operatorname{dim} A=1$.

Proof. (i) By the assumption, there is a subalgebra $K$ of $L$ such that $L=H \dot{+} K$. Then we have

$$
A=A \cap L=A \cap(H+K)=H+(A \cap K) .
$$


Alg. Struc. Appl. Vol. 5 No. 2 (2018) 15-21.

Since $A \cap K$ is an ideal of $L$ contained in $A$, so $A \cap K=0$ or $A \cap K=A$. If $A \cap K=0$, then $A=H$, that is false. But if $A \cap K=A$, then $H \subseteq A \subseteq K$ and so $H=0$. Therefore $A$ is one-dimensional, as required.

(ii) If $H$ is a one-dimensional subalgebra of $A$, then with a same proof by $(i)$, we have two cases: $A \cap K=0$ or $A \cap K=A$. If $A \cap K=0$, Then $A=H$ and so $A$ is one-dimensional. But if $A \cap K=A$, then $H=0$, that is not true.

Some of the following results were given by Gein and Mukhin [5].

Lemma 2.3. Let $L$ be completely factorisable and $H \leq L$ and let $N$ be an ideal of $L$. Then $H$ and $L / N$ are completely factorisable.

Proof. Straightforward.

Proposition 2.4. If $L$ is completely factorisable, then $L$ is $\varphi$-free.

Proof. Since $\varphi(L)$ is permutably complemented in $L$, there is a subalgebra $H$ of $L$ with $L=$ $\varphi(L) \dot{+} H$. But by definition of $\varphi(L)$, we have $\varphi(L) \subseteq H$, forcing $\varphi(L)=0$.

Corollary 2.5. If $L$ is completely factorisable, then $L$ is elementary.

Proof. This follows immediately from Lemma 2.3 and Proposition 2.4.

The following proposition is proved using, step by step, similar arguments as in the proof of [12; Lemma 4], so we will omit its proof.

Proposition 2.6. Let $A$ be a minimal abelian ideal of a Lie algebra $L$. Then $L$ is completely factorisable if and only if there is a subalgebra $B$ of $L$ such that $L=A+B$ and $B$ is completely factorisable.

Proposition 7.9 in [10] together with the above proposition immediately imply the following:

Corollary 2.7. If a solvable Lie algebra $L$ is $\varphi$-free, then $L$ is completely factorisable. 


\section{The main results}

In [I], it is announced that a finite group $G$ is supersolvable if and only if there is a normal subgroup $N$ of $G$ such that $G / N$ is supersolvable and all minimal subgroups of $N$ are complemented in $G$. In the following, we prove a similar result for the case of Lie algebras.

Theorem 3.1. A Lie algebra $L$ is supersolvable if and only if there is a solvable ideal $K$ of $L$ such that $L / K$ is supersolvable and all one-dimensional subalgebras of $K$ have permutable complements in $L$.

Proof. The necessity of the condition is easily established, because any supersolvable Lie algebra contains an ideal of dimension one.

We now prove the sufficiency of the condition. Assume that the theorem is false, and $L$ is a counter-example of minimal dimension. It is obvious that $L$ can not be simple. If $H$ is a proper subalgebra of $L$, then $H \cap K$ is a solvable ideal of $H$ and the factor Lie algebra $H / H \cap K$ is isomorphic to a subalgebra of $L / K$, and so it is supersolvable. Moreover, by Lemma 2.1(i), every one-dimensional subalgebra of $H \cap K$ is permutably complemented in $H$. The minimal choice of $L$ implies that $H$ is supersolvable. Then, invoking [14; Theorem 4.1], L is solvable and all of whose proper subalgebras are abelian. It therefore follows from [14; Theorem 3.6] that $N(L)$ is the unique minimal abelian ideal of $L$ and consequently $N(L) \subseteq K$. We now conclude from the assumption and Lemma 2.2 that $\operatorname{dim}(N(L))=1$, whence $L$ is supersolvable. This contradiction establishes the result.

As a straightforward corollary of Theorem 3.1, we have

Corollary 3.2. If $L$ is a solvable Lie algebra, in which all one-dimensional subalgebras of $L^{2}$ are permutably complemented in $L$, then $L$ is supersolvable.

Theorem 3.3. Let $K$ be a solvable ideal of a Lie algebra $L$ such that $L / K$ is supersolvable. If all maximal subalgebras of every maximal nilpotent subalgebra of $K$ have permutable complements in $L$, then $L$ is supersolvable.

Proof. Assume the result is false and let $L$ be a minimal counterexample. Then

(1) If $N$ is a non-zero ideal of $L$ contained in $K$, then $L / N$ is supersolvable.

Suppose that $\operatorname{dim}(L / N)>1$, and $U / N$ is a maximal nilpotent subalgebra of $K / N$ and $H / N$ is a maximal subalgebra of $U / N$. We consider the following two cases:

Case 1. $N$ is contained in $\varphi(U)$. Then $U / \varphi(U)$ is nilpotent and consequently $U$ is maximal nilpotent subalgebra of $K$, thanks to Barnes [2]. By the assumption, $H$ is permutably complemented in $L$ and then, according to Lemma 2.1(ii), $H / N$ is permutably complemented in $L / N$. 
Alg. Struc. Appl. Vol. 5 No. 2 (2018) 15-21.

Case 2. $N$ is not contained in $\varphi(U)$. Then $U=N+M$ for some maximal subalgebra $M$ of $U$. We choose the subalgebra $D$ to be minimal with respect to $U=N+D$.

It follows from [10; Lemma 7.1] that $N \cap D<\varphi(D)$. However, $D /(N \cap D) \cong U / N$ is nilpotent, implying that $D$ is nilpotent. We consider the subalgebra $V$ of $U$ to be a maximal element in the (non-empty) collection of nilpotent subalgrbras, $D$ say, such that $U=N+D$. It is readily checked that $V$ is a maximal nilpotent subalgebra of $K$ and moreover, $H=H \cap(N+V)=N+(H \cap V)=N+A$ in which $A$ is a maximal subalgebra of $V$ with $H \cap V \subseteq A$. Thus, by hypothesis, there is a subalgebra $B$ of $L$ such that $L=A \dot{+} B$ and then $L / N=(A+N) / N \dot{+}(B+N) / N$. Therefore the Lie algebra $L / N$ together with ideal $K / N$ satisfies the hypothesis of the theorem and hence $L / N$ is supersolvable.

(2) $\operatorname{dim}(N(K))=1$.

It is obvious that $K \cap \varphi(L)=0$; For, otherwise, $L / K \cap \varphi(L)$ and then $L$ are supersolvable, contradicting our supposition. So $N(K)$ is the direct sum of minimal abelian ideals of $L$ which are contained in $K$, by [9; Proposition 2.7]. Since the class of all supersolvable Lie algebras is a saturated formation, we may assume that $N(K)$ is a minimal abelian ideal of $L$. We can easily show that $N(K)$ is a maximal nilpotent subalgebra of $K$. Let $M$ be a maximal subalgebra of $N(K)$. Then there exists a subalgebra $C$ of $L$ such that $L=M \dot{+} C$ and we have $\operatorname{dim} N(K)=1$, thanks to Lemma 2.2. Since $L / N(K)$ is supersolvable by the step (1), so $L$ is supersolvable, a contradiction. This complete the proof of theorem.

As a conseqence of the above theorem, we have

Corollary 3.4. Let $L$ be a solvable Lie algebra. If all maximal subalgebras of every maximal nilpotent subalgebra of $L$ are permutably complemented in $L$, then $L$ is supersolvable.

In the following, we establish, under some conditions, that Theorem 3.3 holds with 'maximal nilpotent subalgebras' replaced by 'the largest nilpotent ideal', which is much easier to find.

Theorem 3.5. Let $L$ be a solvable Lie algebra. Then $L$ is supersolvable if and only if there is an ideal $K$ of $L$ such that $L / K$ is supersolvable and all maximal subalgebras of $N(K)$ have permutable complements in $L$.

Proof. We first prove the sufficiency of the condition. We use induction on the dimension of $L$. We distinguish two cases:

Case 1: $\varphi(L) \neq 0$. If $K \cap \varphi(L)$ is non-zero, then $\operatorname{dim}(L /(K \cap \varphi(L)))<\operatorname{dim}(L)$ and by [9; Proposition 2.6], any maximal subalgebra of $N(K /(K \cap \varphi(L)))$ may be regarded as $M /(K \cap$ $\varphi(L)$ ), in which $M$ is a maximal subalgebra of $N(K)$. By hypothesis, $M$ is permutably 
complemented in $L$ and so $M /(K \cap \varphi(L))$ is permutably complemented in $L /(K \cap \varphi(L))$ because of Lemma 2.1(ii). Hence, by applying induction, it is inferred that the factor Lie algebra $L /(K \cap \varphi(L))$ and then, by [2; Theorem 7], $L$ are supersolvable. We now assume that $K \cap \varphi(L)=0$. Then there is a minimal abelian ideal $N$ of $L$ such that $N \subseteq \varphi(L)$ and $K \cap N=0$. If $L=K+N$, then [10; Lemma 2.1] yields that $L=K$ and consequently $\varphi(L)=0$, a contradiction. So $K+N$ is a proper ideal of $L$. As $N((K+N) / N) \cong N(K) \cong(N(K)+N) / N$, any maximal subalgebra of $N((K+N) / N)$ may be regarded as $(M+N) / N$, where $M$ is a maximal subalgebra of $N(K)$. Noting that $N \subseteq \varphi(L) \subseteq M$. Using the assumption of theorem, [9; Proposition 2.6] and the induction hypothesis, we again deduce that $L / N$ and then $L$ are supersolvable.

Case 2: $\varphi(L)=0$. By Corollary 2.7, $L$ is completely factorisable and hence, by using hypothesis of induction, every proper subalgebra of $L$ is supersolvable. Suppose that $N$ is a minimal abelian ideal of $L$. Then $L=M \dot{+} N$ for some maximal subalgebra $M$ of $L$. But this follows that $L / N$ is supersolvable. By using Lemma 2.2, we therefore conclude that $L$ is supersolvable.

The necessity of the condition is easy established, for any supersolvable Lie algebra contains an ideal of dimension one.

From the above theorem and [4; Corollary 2.6.3], the following corollary is immediate.

Corollary 3.6. Let $L$ be a solvable Lie algebra. If all maximal subalgebras of $L^{2}$ are permutably complemented in $L$, then $L$ is supersolvable.

Theorem 3.7. Let $L$ be a solvable Lie algebra. Then $L$ is supersolvable if and only if there is an ideal $K$ of $L$ such that $L / K$ is supersolvable and all one-dimensional subalgebras of $N(K)$ have permutable complements in $L$.

Proof. The necessity of the condition holds trivially.

Conversely, if $K \cap \varphi(L) \neq 0$ then $\operatorname{dim}(L /(K \cap \varphi(L)))<\operatorname{dim}(L)$ and also, the factor Lie algebra $L /(K \cap \varphi(L))$ satisfies the hypothesis of the theorem thanks to Lemma 2.1. By applying induction and [2; Theorem 7], one deduces that $L /(K \cap \varphi(L))$ and hence $L$ are supersolvable. We now assume that $K \cap \varphi(L)=0$. According to [9; Proposition 2.7], $N(K)=B_{1} \oplus \ldots \oplus B_{n}$ in which the $B_{i}, i=1, \ldots, n$, are minimal abelian ideals of $L$. Since, by the assumption and Lemma 2.2, any ideal $B_{i}$ is one-dimensional, we conclude that any maximal subalgebra of $N(K)$ is ideal in $L$. The result therefore follows from [9; Theorem 3.3].

The following corollary is an immediate consequence of above theorem. 
Alg. Struc. Appl. Vol. 5 No. 2 (2018) 15-21.

Corollary 3.8. Let $L$ be a solvable Lie algebra in which all one-dimensional subalgebras of $N(L)$ have permutable complements. Then $L$ is supersolvable.

\section{ACKNowledgments}

The author would like to thank the referees and Professor A.R. Salemkar for their valuable suggestions, which contributed to improvement of the paper.

\section{REFERENCES}

[1] A. Ballester-Bolinches and X. Guo, On complemented subgroups of finite groups, Arch. Math. Vol. 72 No. 3 (1999), pp. 161-166.

[2] D.W. Barnes, On the cohomology of soluble Lie algebras, Math. Z. Vol. 101 (1967), pp. 343-349.

[3] C. Christensen, Complementation in groups, Math. Z. Vol. 84 (1964), pp. 52-69.

[4] W.A. Graaf, Lie algebras: Theory and Algorithms, North-Holland Mathematical Library, 56, Amsterdam, Elsevier Science, (2000).

[5] A.G. Gein and Yu.N. Mukhin, Complements to subalgebras of Lie algebras, Ural. Gos. Univ. Mat. Zap. Vol. 12 No. 2 (1980), pp. 24-48.

[6] P. Hall, Complemented groups, J. Lond. Math. Soc. Vol 12 (1937), pp. 201-204.

[7] L.C. Kappe and J. Kirtland, Finite groups with trivial Frattini subgroup, Arch. Math. Vol. 80 (2003), pp. $225-234$.

[8] B. Kolman, Relatively complemented Lie algebras, J. Sci. Hiroshima Univ. Ser. A-I Vol 31 (1967), pp. 1-11.

[9] A.R. Salemkar, S. Chehrazi and F. Tayanloo, Characterizations for supersolvable Lie algebras, Comm. Alg. Vol. 41 (2013), pp. 2310-2316.

[10] D.A. Towers, A Frattini theory for algebras, Proc. London Math. Soc. Vol. 27 (1973), pp. 440-462.

[11] D.A. Towers, Elementary Lie algebras, J. London Math. Soc. Vol. 7 (1973), pp. 295-302.

[12] D.A. Towers, On complemented Lie algebras, J. London Math. Soc. Vol. 22 (1980), pp. 63-65.

[13] D.A. Towers, On Lie algebras in which modular pairs of subalgebras are permutable, J. Algebra Vol. 68 No. 2 (1981), pp. 369-377.

[14] D.A. Towers, Minimal non-supersolvable Lie algebras, Algebra, Groups and Geometries Vol 2 (1985), pp. $1-9$.

[15] D.A. Towers, C-Supplemented subalgebras of Lie algebras, J. Lie Theory Vol. 18 No. 3 (2008), pp. $717-724$.

\section{Leila Goudarzi}

Department of mathematics,

University of Ayatollah Alozma Boroujerdi,

Boroujerd, Iran.

le.goudarzi@abru.ac.ir 